\title{
Neural network dynamics underlying gamma synchronization deficits in schizophrenia
}

Running title: Gamma oscillatory dynamics in schizophrenia (43/50 characters)

Daisuke Koshiyama, M.D., Ph.D. ${ }^{1}$; Makoto Miyakoshi, Ph.D.'; Yash B. Joshi, M.D., Ph.D. ${ }^{1}$; Juan L. Molina, M.D. ${ }^{1}$; Kumiko Tanaka-Koshiyama, M.D. ${ }^{1}$; Joyce Sprock, B.A. ${ }^{1}$; David L. Braff, M.D. ${ }^{1}$; Neal R. Swerdlow, M.D., Ph.D. ${ }^{1}$; Gregory A. Light, Ph.D. ${ }^{1,3}$

1. Department of Psychiatry, University of California San Diego, La Jolla, CA, USA 92093-0804

2. Swartz Center for Neural Computation, University of California San Diego, La Jolla, CA, USA 92093-0559

3. VISN-22 Mental Illness, Research, Education and Clinical Center (MIRECC), VA San Diego Healthcare System, San Diego, CA, USA 92161

\section{Corresponding Author:}

Gregory A. Light, Ph.D.

Department of Psychiatry, University of California San Diego

Mail Code: 0804

9500 Gilman Drive

La Jolla, CA, USA 92093-0804

Tel.: (619) 543-2496

Email: glight@health.ucsd.edu

Number of words in abstract: $234 / 250$ words

Number of text body: 2779

Number of words in manuscript: 3367 [including the abstract (234), text body (2779),

figure legends (297 words), and acknowledgments (57 words)]

Number of figures and tables: 5

Number of supplementary files: 13

Number of references: 74 


\section{Abstract}

Gamma band (40-Hz) activity is associated with many sensory and cognitive functions, and is critical for cortico-cortical transmission and the integration of information across neural networks. The capacity to support gamma band activity can be indexed by the auditory steady-state response (ASSR); schizophrenia patients have selectively reduced synchrony to $40-\mathrm{Hz}$ stimulation. While $40-\mathrm{Hz}$ ASSR is a translatable electroencephalographic biomarker with emerging utility for therapeutic development for neuropsychiatric disorders, the spatiotemporal dynamics underlying the ASSR have not yet been characterized. In this study, a novel Granger causality analysis was applied to assess the propagation of gamma oscillations in response to $40-\mathrm{Hz}$ steady-state stimulation across cortical sources in schizophrenia patients $(n=426)$ and healthy comparison subjects $(n=293)$. Results revealed distinct, hierarchically sequenced temporal and spatial response dynamics underlying gamma synchronization deficits in patients. During the response onset interval, patients exhibited abnormal connectivity of superior temporal and frontal gyri, followed by decreased information flow from superior temporal to middle cingulate gyrus. In the later (300-500 ms) interval of the ASSR response, patients showed significantly increased connectivity from superior temporal to middle frontal gyrus followed by broad failures to engage multiple prefrontal brain regions. In conclusion, these findings reveal the rapid disorganization of neural circuit functioning in response to simple gamma-frequency stimulation in schizophrenia patients. Deficits in the generation and maintenance of gamma-band oscillations in schizophrenia reflect a fundamental connectivity abnormality across a distributed network of temporofrontal networks. 
Keywords: gamma-band auditory steady-state response; effective connectivity; source level analysis; schizophrenia; neural temporal dynamics.

\section{Introduction}

Information processing and psychophysiological measures have a long history in characterizing fundamental schizophrenia deficits underlying real word functioning. ${ }^{1-4}$ Recent advances in neuroscience have illuminated the time-linked mechanisms by which anatomically distinct brain regions communicate in order to integrate and coordinate perception, information processing, and cognition in mammals. In particular, the synchronous neural oscillations in the $30-50 \mathrm{~Hz}$ range that occur in the cortex, centered near $40 \mathrm{~Hz}$ and referred to as the "gamma band," appear to reflect a fundamental central nervous system (CNS) resonance frequency critical for communication across multiple brain regions, allowing both basic and complex cognitive information processing to occur. $^{5-11}$

Abnormalities in synchronous neural oscillations also help to explain cognitive deficits in complex brain illnesses like schizophrenia. ${ }^{12-14}$ Schizophrenia is characterized by information processing deficits in addition to a wide range of molecular, neurophysiological and neuroanatomic deficits. In schizophrenia, impairments in transient gamma synchrony measured by electroencephalography (EEG) are linked to not only basic and higher order cognitive deficits, but also clinical symptoms ${ }^{15-18}$; therefore, in schizophrenia, gamma band deficits are hypothesized to reflect the fundamental problems with "timing or sequencing component of mental activity" that lead to a heterogeneous constellation of clinical and cognitive deficits that characterize schizophrenia. ${ }^{19}$ 
The capacity to support the generation of synchronous neural activity can also be more directly interrogated by using paradigms that aim to "entrain" oscillations at particular frequencies. For example, studies of healthy subjects have revealed that when receiving periodic stimulation, neural networks behave like tuned oscillators, with EEG synchronizing to the frequency of stimulation. ${ }^{20-22}$ Schizophrenia patients have selectively reduced synchrony to auditory-based 40-Hz stimulation, but normal responses to other rates of stimulation. ${ }^{3,18,23-36}$ This gamma band auditory-steady response (ASSR) has received significant attention as a potential translatable biomarker advancing procognitive treatment development in schizophrenia and other brain disorders, such as bipolar disorder, autism spectrum disorder and 22q11.2 deletion syndrome. ${ }^{26,35,37-44}$

Despite this interest, there is limited information in schizophrenia patients about how different brain regions coordinate, interact and produce ASSR deficits through the stimulus entrainment period. ${ }^{45,46}$ Reports suggest the time course of ASSR may reflect distinct aspects of schizophrenia pathophysiology. Specifically, the 40-Hz ASSR is usually measured across a full $500 \mathrm{~ms}$ stimulation interval, with an early $\sim 1-200 \mathrm{~ms}$ stimulus onset interval, and late $\sim 300-500 \mathrm{~ms}$ maintenance interval ${ }^{27}$, 28: both firstepisode as well as chronic schizophrenia patients have gamma-band ASSR deficits in early and later time intervals, but individuals at clinical high-risk for psychosis, showed deficits in only the later maintenance time interval. ${ }^{25,27,28}$

Augmenting magnetic resonance imaging (MRI)-based studies of structural connectivity, gamma-band ASSR can leverage the functional fine temporal resolution of EEG to assess effective connectivity, i.e. the causal interactions (i.e. directed information flows) among underlying brain regions that generate and maintain $40-\mathrm{Hz}$ ASSR. This study aimed to use a novel, data-driven Granger causality analysis to deconstruct the time 
course and patterns of effective connectivity of cortical sources underlying the $40-\mathrm{Hz}$ ASSR and its deficits in schizophrenia patients. This Granger causality approach enables an analysis of network dynamics with directed information flow using a correlation with time delay.

\section{Methods}

\section{Subjects}

Participants were 426 schizophrenia patients and 293 healthy comparison subjects (Table 1). Patients were recruited from community residential facilities and via clinician referral, and diagnosed using a modified version of the Structured Clinical Interview for DSMIV-TR. Antipsychotic medications were prescribed for 388 schizophrenia patients. Healthy comparison subjects were recruited through internet advertisements. Exclusion criteria included an inability to understand the consent processes and/or provide consent or assent, not being a fluent English speaker, previous significant head injury with loss of consciousness, neurologic illness or severe systemic illness. Written informed consent was obtained from each subject. Audiometric testing was used to ensure that all participants could detect $1000-\mathrm{Hz}$ tones at $40 \mathrm{~dB}$. The Institutional Review Board of University of California San Diego approved all experimental procedures (071128, 071831, 170147). The current study was conducted in accordance with the Declaration of Helsinki.

\section{Stimuli and procedures}

Auditory steady-state stimuli were presented to subjects by means of foam insert earphones (Model 3A; Aearo Company Auditory Systems, Indianapolis, Indiana). The 
stimuli were 1-millisecond, 93-dB clicks presented at $40 \mathrm{~Hz}$ in 500-millisecond trains. A block typically contained 200 trains of the clicks with 500-millisecond intervals. During the session, participants watched a silent cartoon video.

\section{Electroencephalography recording and preprocessing}

EEG data were continuously digitized at a rate of $1000 \mathrm{~Hz}$ (nose reference, forehead ground) using a 40-channel Neuroscan system (Neuroscan Laboratories, El Paso, Texas). The electrode montage was based on standard positions in the International 10-5 electrode system ${ }^{47}$ fit to the MNI template head used in EEGLAB, including AFp10 and AFp9 as horizontal EOG channels, IEOG and SEOG above and below the left eye as vertical EOG channels, Fp1, Fp2, F7, F8, Fz, F3, F4, FC1, FC2, FC5, FC6, C3, Cz, C4, CP1, CP2, CP5, CP6, P7, P3, Pz, P4, P8, T7, T8, TP9, TP10, FT9, FT10, PO9, PO10, O1, O2, and Iz. Electrode-to-skin impedance mediated by conductive gel was brought below $4 \mathrm{k} \Omega$. The system acquisition band pass was 0.5-100 Hz. Offline, EEG data were imported to EEGLAB 14.1.2 ${ }^{48}$ running under Matlab 2017b (The MathWorks, Natick, MA). Data were high-pass filtered (FIR, Hamming window, cutoff frequency $0.5 \mathrm{~Hz}$, transition bandwidth 0.5). EEGLAB plugin clean_rawdata() including Artifact Subspace Reconstruction was applied to reduce high-amplitude artifacts. ${ }^{49-54}$ The parameters used were: flat line removal, $10 \mathrm{~s}$; electrode correlation, 0.7; ASR, 20; window rejection, 0.5. Mean channel rejection rate was $4.2 \%$ (SD 2.3, range 0-15.8). Mean data rejection rate was $2.0 \%$ (SD 3.5, range 0-22.4). The rejected channels were interpolated using EEGLAB's spline interpolation function. Data were re-referenced to average. Adaptive mixture ICA ${ }^{55}$ was applied to the preprocessed scalp recording data to obtain temporally maximally independent components (ICs). For scalp topography of each IC derived, 
equivalent current dipole was estimated using Fieldtrip functions. ${ }^{56}$ For scalp topographies more suitable for symmetrical bilateral dipoles, two symmetrical dipoles were estimated. ${ }^{57}$ To select brain ICs among all types of ICs, EEGLAB plugin ICLabel() was used. ${ }^{58}$ The inclusion criteria were 1) 'brain' label probability $>0.7$ and 2) residual variance i.e., $\operatorname{var}(($ actual scalp topography) - (theoretical scalp projection from the fitted dipole) $) /$ var(actual scalp topography) $<0.15$. Continuous EEG data were segmented into epochs that started at $-250 \mathrm{~ms}$ and end at $750 \mathrm{~ms}$ relative to stimulus onset. Epoch rejection was performed for each participant using their median value of single-trial power spectral density (PSD) of remaining ICs. The single-trial PSD values were calculated and the median value across trials was subtracted. The residual errors from the median were z-scored. Any epoch with mean value $>2$ between 15 to $35 \mathrm{~Hz}$ was rejected as contaminated by muscle potentials. The final processed data had a mean of 158.6 trials (SD 18.4).

\section{Effective connectivity analyses}

To calculate grand-mean effective connectivity across ICs for each group, we applied EEGLAB plugin groupSIFT, which recently demonstrated successful application in other clinical EEG project. ${ }^{59}$ Using the same set of 9324 ICs in total preselected as representative brain ICs (mean 13.0 ICs per person, SD 4.4, range 4-28), time-frequency decomposed renormalized partial directed coherence (RPDC) ${ }^{60}$ was calculated across ICs (sliding window length $0.3 \mathrm{~s}$, window step size $4 \mathrm{~ms}$, logarithmically distributing 50 frequency bins from 2 to $55 \mathrm{~Hz}$, baseline period -0.1 to $0 \mathrm{~s}$ ). This generated a connectivity matrix with the dimension of IC $\times$ IC for each individual. The grand-average optimum model order was 11.6 (SD 1.6) i.e. delayed effective connectivity up to about $48 \mathrm{~ms}$ was 
utilized. The estimated equivalent dipole locations of the corresponding ICs were convolved with 3-D Gaussian kernel with $20 \mathrm{~mm}$ FWHM to obtain probabilistic dipole density. The dipole density inside the brain space is segmented into anatomical regions defined by automated anatomical labeling (AAL). ${ }^{61}$ The original AAL has 88 anatomical regions, but those basal and limbic regions that are unlikely to be scalp-measured EEG sources were integrated into two umbrella terms, upper basal and lower basal. The vagueness was intentional so that misleading use of limbic/basal label for EEG sources is avoided.

The original labels 'upper basal' and 'lower basal' were reported as ventral midcingulate, 'mid-cingulate' as dorsal mid-cingulate, and 'insula' as inferior frontal. This systematic bias toward depth in single-dipole fitting is due to the fact that 'the large spatial extent of the many dipole layers that evidently generate EEG disqualifies them as single dipoles'. ${ }^{62}$ If this is the case, estimating shallower source regions along with radial direction as suggested here should provide a good heuristic correction. Individual IC $\times$ IC connectivity matrix is thus mapped to $76 \times 76$ custom anatomical region matrix, on which RPDC was also mapped as a weighting factor to modulate pairwise dipole density to calculate graph edges. For both groups, including a minimum of $80 \%$ of unique subjects was set to be an inclusion criterion for each graph node to be analyzed in the next stage. Also, for the group comparison, 47/76 graph nodes showed overlap between the groups, which explained $79.3 \%$ of total dipole density. For details of this solution, refer to our previous publication using this method. ${ }^{59}$ The analysis included $35-45 \mathrm{~Hz}$ as the frequency range of interest to be consistent with the frequency range in previous gammaband ASSR studies. ${ }^{27-30}$ For the statistics of time-frequency decomposed RPDC withinand across-groups, weak family-wise error rate control was applied. ${ }^{63,64}$ 


\section{Results}

The connectivity matrix that represents the group-difference of gamma-band ASSR with the initial results ( $p<0.05$, corrected; two-tailed) is shown in Figure 1. A predefined $p$ value threshold of $p<0.0001$ (two-tailed ${ }^{65}$ ) revealed 15 graph edges (Figure 2, Supplementary Figure 1. and Supplementary Movie 1, 2). The matrix and movies of healthy comparison subjects (Supplementary Figure 2 and Supplementary Movie 3, 4) and schizophrenia patients (Supplementary Figure 3 and Supplementary Movie 5, 6) are shown in supplementary information.

\section{Neural network dynamics}

Based on the temporal dynamics, spatial interactions reflecting neural network dynamics underlying gamma synchronization deficits in patients are shown in Figure 3A. This network figure shows rank order latency of each information flow along with Figure 3B, revealing that the abnormal neural network centroid moves from an early local network centered at the right superior temporal to a late network centered at the left prefrontal in schizophrenia patients. Shortly after the response onset, a significant increase in connectivity was observed between temporal and frontal regions in schizophrenia patients (8 in Figure 3B), followed by decreased connectivity abnormalities within prefrontal regions (10, 12, 13 in Figure 3B), described below.

\section{Early ASSR time interval: network centered at superior temporal gyrus}

Schizophrenia patients showed abnormal connectivity during the onset interval of ASSR (i.e., 1-200 ms). Specifically, patients showed increased connectivity between right 
superior temporal and right inferior frontal gyri (Figure 2A and Figure 3B). Following response onset, information flow from the right superior temporal to the ventral middlecingulate was decreased in patients compared to healthy comparison subjects $(\sim 100 \mathrm{~ms}$ to $300-400 \mathrm{~ms}$ ). Patients also showed large increases in information flow from the right supplementary motor area to the left inferior frontal region compared to healthy comparison subjects.

\section{Middle time interval: information flow from inferior frontal to occipital regions}

In the 200-300 ms response interval, connections from the right ventral middle cingulate to the right occipital regions, (i.e., right medial occipital and the right calcarine, right inferior frontal to the right medial occipital) were decreased in schizophrenia patients compared to healthy comparison subjects. These reductions continue into the later interval of ASSR (Figure 2B and Figure 3B).

\section{Later time interval: network centered at prefrontal regions}

Schizophrenia related decreased connectivity during this later phase of ASSR (i.e., 300$500 \mathrm{~ms}$ ) included the flows from the right parietal (supramarginal) to the left inferior frontal regions; this time interval was characterized by prominent and broad reductions in connectivity across prefrontal regions in schizophrenia patients (Figure 2C and Figure 3B).

\section{Discussion}

Results of this study provide evidence of abnormal neural network dynamics underlying gamma synchronization deficits in schizophrenia patients compared to healthy 
comparison subjects. A novel Granger causality analysis was applied to EEG recordings obtained from large cohorts of schizophrenia patients and healthy comparison subjects ${ }^{66}$. Gamma-band ASSR deficits in schizophrenia patients reflect abnormal neural network functioning centered at the superior temporal gyrus at the onset of the auditory steadystate response that then cascade "forward" and yield widespread abnormalities in the engagement of prefrontal brain regions in the later intervals of the response (Figure 4). Remarkably, before these decreases, abnormally increased information flows were evident in the local neural networks in patients (Figure 3B).

These results are strikingly consistent with a previous ECoG study by Tada et al., ${ }^{46}$ which showed cortical surface levels of gamma-band ASSR in humans. They demonstrated prominent increases of gamma oscillations at the right primary auditory cortex (A1), right supramarginal gyrus and right supplementary motor area as well as moderate increases of activity at bilateral prefrontal gyri. While the Tada et al. ${ }^{46}$ findings were from patients undergoing neurosurgical intervention for treatment refractory epilepsy, they nonetheless suggest that these gamma-band locations are critical generators of ASSR in humans, and thus may be loci responsible for abnormalities in schizophrenia or other impaired neuropsychiatric patient populations. ${ }^{46}$ The current study, using novel and non-invasive EEG connectivity analyses may thus fill a gap between this study of patients with schizophrenia and the study of Tada et al. using ECoG and potentially may converge with findings from animal models. ${ }^{46,67}$

The present study supports the increasing use of temporally distinct ASSR time intervals in studies of patients with psychosis. In this context, early and later time intervals of gamma oscillation appear to reflect dissociable response intervals. ${ }^{25,27,28}$ According to the prior studies, while first-episode schizophrenia patients showed gamma-band ASSR 
deficits both in the early and later intervals, individuals with clinical high-risks for psychosis showed reductions only in the later time interval. The local neural network centered at the superior temporal gyrus in the early onset time interval may be preserved in individuals with clinical high-risks for psychosis, consistent with the progressive volume reduction within the superior temporal gyrus observed after the onset of schizophrenia in first-episode schizophrenia patients. ${ }^{68,69}$ The observed prefrontal gamma connectivity decrease during the later time interval in schizophrenia patients may reflect severe structural alterations in both gray and white matter. ${ }^{70-73}$

Support for the use of distinct time intervals also comes from an experimental medicine trial which revealed distinct pharmacodynamic results over the later maintenance, but not early onset phase of ASSR response in both healthy subjects and schizophrenia patients. Specifically, we reported that a single dose of the non-competitive N-methyl-D-aspartate (NMDA) receptor modulator, memantine, robustly increased (i.e., normalized) ASSR over later time intervals in schizophrenia patients. ${ }^{37}$

Results of this study should be considered in the context of some limitations. First, this is a cross-sectional cohort study of a heterogeneous sample of schizophrenia patients, the majority of whom were receiving complex medication regimens. As is the case for most large-scale studies of schizophrenia patients, the medication, psychosocial environments, and other important factors that could potentially influence brain function were not experimentally controlled. Second, the schizophrenia patients in this study had a well-established illness; results therefore may not generalize to at risk or early-illness psychosis patients. Third, 40 EEG channels were used for connectivity analyses. Future studies may fruitfully use higher density recordings with at least 64 channels, ${ }^{74}$ individual MRI data, and digitized scalp sensor locations rather than template head models and 
reliance on standardized electrode locations for potentially improved accuracy of source dynamics. Fourth, effective connectivity analyses are descriptive; they do not tell us about the functional roles of observed connectivity patterns or abnormalities; future studies will be needed to show their precise functional roles of the connectivity in neural substrates in patients with schizophrenia.

In conclusion, this study characterizes the disorganized propagation of gamma frequency oscillations in real-time in schizophrenia patients by applying a novel multivariate Granger causality analysis to large-scale scalp EEG datasets. Our findings provide evidence that relatively circumscribed abnormalities in the processing of simple 40-Hz auditory stimuli at the superior temporal gyrus in schizophrenia patients rapidly cascade forward to produce widespread failures to engage distributed prefrontal brain regions that are necessary to support gamma oscillations. Deficits in the generation and maintenance of stimulus-driven gamma-band oscillations reflect a fundamental connectivity abnormality across a distributed network of temporal and frontal regions. Clarification of the neural mechanisms underlying the networks detected in this study, in both future clinical and animal studies, will strengthen the utility of gamma-band ASSR as a translatable brain marker; this should further pave the way for clarifying the pathophysiology of neuropsychiatric and neurological diseases and the development of novel drug and behavioral therapeutic interventions. 


\section{Acknowledgements}

This study was supported by JSPS Overseas Research Fellowships (D. Koshiyama). Swartz Center for Computational Neuroscience is supported by generous gift of Swartz Foundation (New York; M. Miyakoshi). The funders had no role in the study design, data collection and analysis, publication decision, or manuscript preparation.

\section{Conflict of Interest}

The authors declare no conflict of interest. 


\section{References}

1. Braff D, Stone C, Callaway E, et al. Prestimulus effects on human startle reflex in normals and schizophrenics. Psychophysiology 1978;15:339-343.

2. Braff DL. Information processing and attention dysfunctions in schizophrenia. Schizophr Bull 1993;19:233-259.

3. Kwon JS, O'Donnell BF, Wallenstein GV, et al. Gamma frequency-range abnormalities to auditory stimulation in schizophrenia. Arch Gen Psychiatry 1999;56:1001-1005.

4. Light GA, Braff DL. Mismatch negativity deficits are associated with poor functioning in schizophrenia patients. Arch Gen Psychiatry 2005;62:127-136.

5. Joliot M, Ribary U, Llinas R. Human oscillatory brain activity near $40 \mathrm{~Hz}$ coexists with cognitive temporal binding. Proc Natl Acad Sci U S A 1994;91:11748-11751.

6. Traub RD, Whittington MA, Stanford IM, Jefferys JG. A mechanism for generation of long-range synchronous fast oscillations in the cortex. Nature 1996;383:621-624.

7. Miltner WH, Braun C, Arnold M, Witte H, Taub E. Coherence of gamma-band EEG activity as a basis for associative learning. Nature 1999;397:434-436.

8. Rodriguez E, George N, Lachaux JP, et al. Perception's shadow: long-distance synchronization of human brain activity. Nature 1999;397:430-433.

9. Hagoort P, Hald L, Bastiaansen M, Petersson KM. Integration of word meaning and world knowledge in language comprehension. Science 2004;304:438-441.

10. Spellman T, Rigotti M, Ahmari SE, et al. Hippocampal-prefrontal input supports spatial encoding in working memory. Nature 2015;522:309-314.

11. Galuske RAW, Munk MHJ, Singer W. Relation between gamma oscillations and neuronal plasticity in the visual cortex. Proc Natl Acad Sci US A 2019;116:2331723325.

12. Sun Y, Farzan F, Barr MS, et al. gamma oscillations in schizophrenia: mechanisms and clinical significance. Brain Res 2011;1413:98-114.

13. Gandal MJ, Edgar JC, Klook K, Siegel SJ. Gamma synchrony: towards a translational biomarker for the treatment-resistant symptoms of schizophrenia. Neuropharmacology 2012;62:1504-1518.

14. Uhlhaas PJ, Singer W. Oscillations and neuronal dynamics in schizophrenia: the search for basic symptoms and translational opportunities. Biol Psychiatry 2015;77:1001-1009.

15. Spencer KM, Nestor PG, Perlmutter R, et al. Neural synchrony indexes disordered perception and cognition in schizophrenia. Proc Natl Acad Sci $U S A$ 
2004; 101:17288-17293.

16. Uhlhaas PJ, Haenschel C, Nikolic D, Singer W. The role of oscillations and synchrony in cortical networks and their putative relevance for the pathophysiology of schizophrenia. Schizophr Bull 2008;34:927-943.

17. Uhlhaas PJ, Singer W. Abnormal neural oscillations and synchrony in schizophrenia. Nat Rev Neurosci 2010;11:100-113.

18. Hirano Y, Oribe N, Kanba S, et al. Spontaneous Gamma Activity in Schizophrenia. JAMA Psychiatry 2015;72:813-821.

19. Andreasen NC. A unitary model of schizophrenia: Bleuler's "fragmented phrene" as schizencephaly. Arch Gen Psychiatry 1999;56:781-787.

20. Galambos R, Makeig S, Talmachoff PJ. A 40-Hz auditory potential recorded from the human scalp. Proc Natl Acad Sci U S A 1981;78:2643-2647.

21. Galambos R, Makeig S. Physiological studies of central masking in man. I: The effects of noise on the $40-\mathrm{Hz}$ steady-state response. $J$ Acoust Soc Am 1992;92:2683-2690.

22. Makeig S. Auditory event-related dynamics of the EEG spectrum and effects of exposure to tones. Electroencephalogr Clin Neurophysiol 1993;86:283-293.

23. Brenner CA, Sporns O, Lysaker PH, O'Donnell BF. EEG synchronization to modulated auditory tones in schizophrenia, schizoaffective disorder, and schizotypal personality disorder. Am J Psychiatry 2003;160:2238-2240.

24. Hamm JP, Bobilev AM, Hayrynen LK, et al. Stimulus train duration but not attention moderates gamma-band entrainment abnormalities in schizophrenia. Schizophr Res 2015;165:97-102.

25. Light GA, Hsu JL, Hsieh $\mathrm{MH}$, et al. Gamma band oscillations reveal neural network cortical coherence dysfunction in schizophrenia patients. Biol Psychiatry 2006;60:1231-1240.

26. Spencer KM, Salisbury DF, Shenton ME, McCarley RW. Gamma-band auditory steady-state responses are impaired in first episode psychosis. Biol Psychiatry 2008;64:369-375.

27. Tada M, Nagai T, Kirihara K, et al. Differential Alterations of Auditory Gamma Oscillatory Responses Between Pre-Onset High-Risk Individuals and FirstEpisode Schizophrenia. Cereb Cortex 2016;26:1027-1035.

28. Koshiyama D, Kirihara K, Tada M, et al. Auditory gamma oscillations predict global symptomatic outcome in the early stages of psychosis: A longitudinal investigation. Clin Neurophysiol 2018;129:2268-2275.

29. Koshiyama D, Kirihara K, Tada M, et al. Electrophysiological evidence for 
abnormal glutamate-GABA association following psychosis onset. Transl Psychiatry 2018;8:211.

30. Koshiyama D, Kirihara K, Tada M, et al. Gamma-band auditory steady-state response is associated with plasma levels of d-serine in schizophrenia: An exploratory study. Schizophr Res 2019;208:467-469.

31. Edgar JC, Chen YH, Lanza M, et al. Cortical thickness as a contributor to abnormal oscillations in schizophrenia? Neuroimage Clin 2014;4:122-129.

32. Teale P, Collins D, Maharajh K, et al. Cortical source estimates of gamma band amplitude and phase are different in schizophrenia. Neuroimage 2008;42:14811489.

33. Tsuchimoto R, Kanba S, Hirano S, et al. Reduced high and low frequency gamma synchronization in patients with chronic schizophrenia. Schizophr Res 2011;133:99-105.

34. Vierling-Claassen D, Siekmeier P, Stufflebeam S, Kopell N. Modeling GABA alterations in schizophrenia: a link between impaired inhibition and altered gamma and beta range auditory entrainment. J Neurophysiol 2008;99:2656-2671.

35. Wilson TW, Hernandez OO, Asherin RM, et al. Cortical gamma generators suggest abnormal auditory circuitry in early-onset psychosis. Cereb Cortex 2008; 18:371-378.

36. Thune H, Recasens M, Uhlhaas PJ. The 40-Hz Auditory Steady-State Response in Patients With Schizophrenia: A Meta-analysis. JAMA Psychiatry 2016;73:1145-1153.

37. Light GA, Zhang W, Joshi YB, Bhakta S. Single-Dose Memantine Improves Cortical Oscillatory Response Dynamics in Patients with Schizophrenia. 2017;42:2633-2639.

38. O'Donnell BF, Hetrick WP, Vohs JL, et al. Neural synchronization deficits to auditory stimulation in bipolar disorder. Neuroreport 2004;15:1369-1372.

39. Reite M, Teale P, Rojas DC, et al. MEG auditory evoked fields suggest altered structural/functional asymmetry in primary but not secondary auditory cortex in bipolar disorder. Bipolar Disord 2009;11:371-381.

40. Rass O, Krishnan G, Brenner CA, et al. Auditory steady state response in bipolar disorder: relation to clinical state, cognitive performance, medication status, and substance disorders. Bipolar Disord 2010;12:793-803.

41. Oda Y, Onitsuka T, Tsuchimoto R, et al. Gamma band neural synchronization deficits for auditory steady state responses in bipolar disorder patients. PLoS One 2012;7:e39955. 
42. Isomura S, Onitsuka $\mathrm{T}$, Tsuchimoto $\mathrm{R}$, et al. Differentiation between major depressive disorder and bipolar disorder by auditory steady-state responses. $J$ Affect Disord 2016;190:800-806.

43. Edgar JC, Fisk CLt, Liu S, et al. Translating Adult Electrophysiology Findings to Younger Patient Populations: Difficulty Measuring 40-Hz Auditory Steady-State Responses in Typically Developing Children and Children with Autism Spectrum Disorder. Dev Neurosci 2016;38:1-14.

44. Larsen KM, Pellegrino G, Birknow MR, et al. 22q11.2 Deletion Syndrome Is Associated With Impaired Auditory Steady-State Gamma Response. Schizophr Bull 2018;44:388-397.

45. Farahani ED, Goossens T, Wouters J, van Wieringen A. Spatiotemporal reconstruction of auditory steady-state responses to acoustic amplitude modulations: Potential sources beyond the auditory pathway. Neuroimage 2017;148:240-253.

46. Tada M, Kirihara K, Ishishita Y, et al. Global and parallel cortical processing of auditory gamma oscillatory responses in humans. 2019. CELL-REPORTS-D-1902197. Available at SSRN: https://ssrn.com/abstract $=3417938$ or http://dx.doi.org/10.2139/ssrn.3417938

47. Oostenveld R, Praamstra P. The five percent electrode system for high-resolution EEG and ERP measurements. Clin Neurophysiol 2001;112:713-719.

48. Delorme A, Makeig S. EEGLAB: an open source toolbox for analysis of singletrial EEG dynamics including independent component analysis. $J$ Neurosci Methods 2004;134:9-21.

49. Kothe CA, Makeig S. BCILAB: a platform for brain-computer interface development. J Neural Eng 2013;10:056014.

50. Mullen TR, Kothe CA, Chi YM, et al. Real-Time Neuroimaging and Cognitive Monitoring Using Wearable Dry EEG. IEEE Trans Biomed Eng 2015;62:25532567.

51. Chang CY, Hsu SH, Pion-Tonachini L, Jung TP. Evaluation of Artifact Subspace Reconstruction for Automatic EEG Artifact Removal. Conf Proc IEEE Eng Med Biol Soc 2018;2018:1242-1245.

52. Gabard-Durnam LJ, Mendez Leal AS, Wilkinson CL, Levin AR. The Harvard Automated Processing Pipeline for Electroencephalography (HAPPE): Standardized Processing Software for Developmental and High-Artifact Data. Front Neurosci 2018;12:97.

53. Blum S, Jacobsen NSJ, Bleichner MG, Debener S. A Riemannian Modification of 
Artifact Subspace Reconstruction for EEG Artifact Handling. Front Hum Neurosci 2019;13:141.

54. Chang CY, Hsu SH, Pion-Tonachini L, Jung TP. Evaluation of Artifact Subspace Reconstruction for Automatic Artifact Components Removal in Multi-channel EEG Recordings. IEEE Trans Biomed Eng 2020;67:1114-1121.

55. Delorme A, Palmer J, Onton J, Oostenveld R, Makeig S. Independent EEG sources are dipolar. PLoS One 2012;7:e30135.

56. Oostenveld R, Fries P, Maris E, Schoffelen JM. FieldTrip: Open source software for advanced analysis of MEG, EEG, and invasive electrophysiological data. Comput Intell Neurosci 2011;2011:156869.

57. Piazza C, Miyakoshi M, Akalin-Acar Z, Cantiani C, Reni G, Bianchi AM. An Automated Function for Identifying EEG Independent Components Representing Bilateral Source Activity. XIV Mediterranean Conference on Medical and Biological Engineering and Computing 2016: Springer International Publishing; 2016:105-109.

58. Pion-Tonachini L, Kreutz-Delgado K, Makeig S. ICLabel: An automated electroencephalographic independent component classifier, dataset, and website. Neuroimage 2019;198:181-197.

59. Loo SK, Miyakoshi M, Tung K, et al. Neural activation and connectivity during cued eye blinks in Chronic Tic Disorders. Neuroimage Clin 2019;24:101956.

60. Schelter B, Timmer J, Eichler M. Assessing the strength of directed influences among neural signals using renormalized partial directed coherence. $J$ Neurosci Methods 2009;179:121-130.

61. Tzourio-Mazoyer N, Landeau B, Papathanassiou D, et al. Automated anatomical labeling of activations in SPM using a macroscopic anatomical parcellation of the MNI MRI single-subject brain. Neuroimage 2002;15:273-289.

62. Nunez P, Srinivasan R. Electric Fields of the Brain. New York, NY: Oxford University Press; 2006.

63. Nichols T, Hayasaka S. Controlling the familywise error rate in functional neuroimaging: a comparative review. Stat Methods Med Res 2003;12:419-446.

64. Groppe DM, Urbach TP, Kutas M. Mass univariate analysis of event-related brain potentials/fields I: a critical tutorial review. Psychophysiology 2011;48:1711-1725.

65. Koshiyama D, Miyakoshi M, Joshi YB, et al. Abnormal effective connectivity underlying auditory mismatch negativity impairments in schizophrenia. Biol Psychiary Cogn Neurosci Neuroimaging In press.

66. Granger CWJ. Investigating Causal Relations by Econometric Models and Cross- 
spectral Methods. Econometrica 1969;37:424-438.

67. Kozono N, Honda S, Tada M, et al. Auditory Steady State Response; nature and utility as a translational science tool. Sci Rep 2019;9:8454.

68. Kasai K, Shenton ME, Salisbury DF, et al. Progressive decrease of left superior temporal gyrus gray matter volume in patients with first-episode schizophrenia. Am J Psychiatry 2003;160:156-164.

69. Kasai K, Shenton ME, Salisbury DF, et al. Progressive decrease of left Heschl gyrus and planum temporale gray matter volume in first-episode schizophrenia: a longitudinal magnetic resonance imaging study. Arch Gen Psychiatry 2003;60:766-775.

70. Kelly S, Jahanshad N, Zalesky A, et al. Widespread white matter microstructural differences in schizophrenia across 4322 individuals: results from the ENIGMA Schizophrenia DTI Working Group. 2018;23:1261-1269.

71. Koshiyama D, Fukunaga M, Okada N, et al. Role of frontal white matter and corpus callosum on social function in schizophrenia. Schizophr Res 2018;202:180-187.

72. van Erp TGM, Walton E, Hibar DP, et al. Cortical Brain Abnormalities in 4474 Individuals With Schizophrenia and 5098 Control Subjects via the Enhancing Neuro Imaging Genetics Through Meta Analysis (ENIGMA) Consortium. Biol Psychiatry 2018;84:644-654.

73. Koshiyama D, Fukunaga M, Okada N, et al. White matter microstructural alterations across four major psychiatric disorders: mega-analysis study in 2937 individuals. Mol Psychiatry 2020;25:883-895.

74. Light GA, Swerdlow NR. Selection criteria for neurophysiologic biomarkers to accelerate the pace of CNS therapeutic development. Neuropsychopharmacology 2020;45:237-238. 


\section{Figure legends}

Figure 1 Connectivity matrix of $76 \times 76$ anatomical region of interests (ROIs) that represents the group-difference

Figure 2 Difference of effective connectivity between schizophrenia and healthy comparison subject groups

Note: Lower row of brain images indicates one frame of the effective connectivity movie at $130 \mathrm{~ms}$ (A), $322 \mathrm{~ms}$ (B) $354 \mathrm{~ms}$ (C) after the stimulus onset seen from an axial view (Supplementary Movie 1 and 2); upper row of brain images indicates main findings of connectivity in the flame; the panel shows the envelope of the significant edges between 35 and $45 \mathrm{~Hz}$; lower part of figure shows the five major connectivity; solid black line at Latency $=0$ is the stimulus onset; broken black line indicates region of interest between 35 and $45 \mathrm{~Hz}$ and between 0 and $500 \mathrm{~ms}$; ' the original label is 'lower basal' (refer to Methods and Materials section).

Abbreviations: RPDC, renormalized partial directed coherence; Inf frontal; inferior frontal; Sup temporal; superior temporal; Supple motor area; supplementary motor area; SZ, schizophrenia; HCS, healthy comparison subject; A, anterior; P, posterior; L, left; R, right.

Figure 3 Neural networks (A) and temporal dynamics (B) underlying gamma oscillation deficits in schizophrenia patients

Note: The numbers indicate rank order latency of each information flow; ${ }^{* 1}$ the original label is 'lower basal'; ${ }^{* 2}$ the original label is 'upper basal' (refer to Methods and Materials section). Abbreviations: Sup frontal; superior frontal; Mid frontal; middle 
frontal; Supple motor, supplementary motor area; Inf frontal, inferior frontal; Dorsal midcingulate; dorsal middle cingulate; Vent mid-cingulate, ventral middle cingulate; Sup temporal; superior temporal; Med occipital, medial occipital; RPDC, renormalized partial directed coherence; L, left; R, right.

Figure 4 Abnormal neural network at the superior temporal gyrus in early phase and those networks at the prefrontal in late phase in schizophrenia patients 
Table 1 Demographic and clinical characteristics of subjects

\begin{tabular}{lllll}
\hline & \multicolumn{2}{l}{$\begin{array}{l}\text { Healthy comparison subjects } \\
(\mathrm{n}=293)\end{array}$} & \multicolumn{2}{l}{$\begin{array}{l}\text { Schizophrenia patients } \\
(\mathrm{n}=426)\end{array}$} \\
& Mean & SD & Mean & SD \\
\hline Gender (male/female) & $141 / 152$ & - & $309 / 117$ & - \\
Age (years) & 44.7 & 11.4 & 45.5 & 9.5 \\
Education (years) & 14.9 & 2.3 & 12.0 & 2.2 \\
Duration of illness (years) $^{\text {a }}$ & - & - & 23.7 & 10.1 \\
SAPS total score $^{\mathrm{b}}$ & - & - & 8.9 & 4.1 \\
SANS total score $^{\mathrm{b}}$ & - & - & 14.3 & 4.3 \\
GAF $^{\mathrm{c}}$ & - & - & 40.9 & 6.6 \\
\hline
\end{tabular}

Note: ${ }^{a}$ Three subjects have no data of duration of illness; ${ }^{b}$ Four subjects have no SAPS or SANS data; ${ }^{\mathrm{c}}$ One subject has no GAF data.

Abbreviations: SAPS, Scale for the Assessment of Positive Symptoms; SANS, Scale for the Assessment of Negative Symptoms. 
Figure 1 Koshiyama et al.

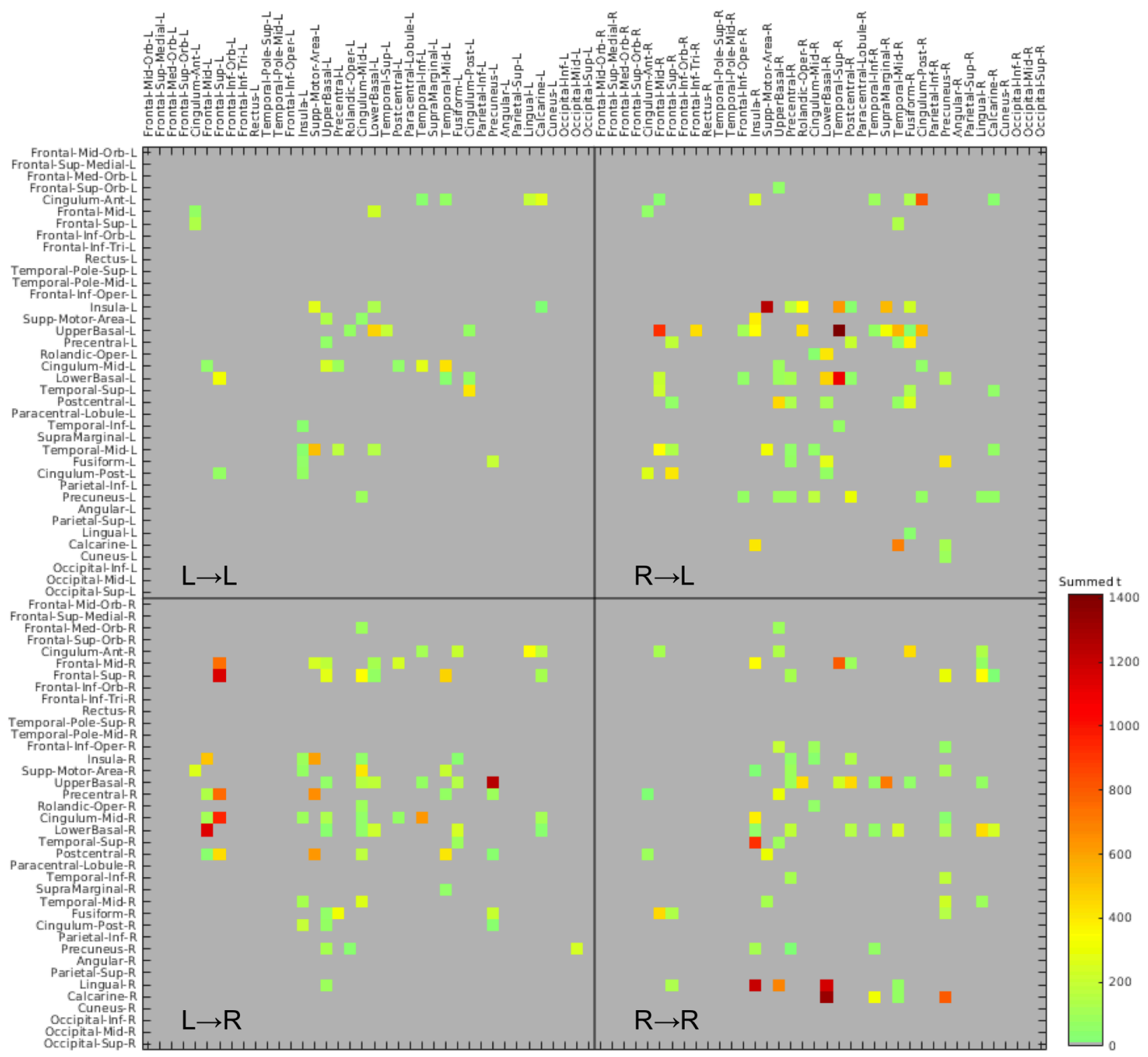


Figure 2 Koshiyama et al.
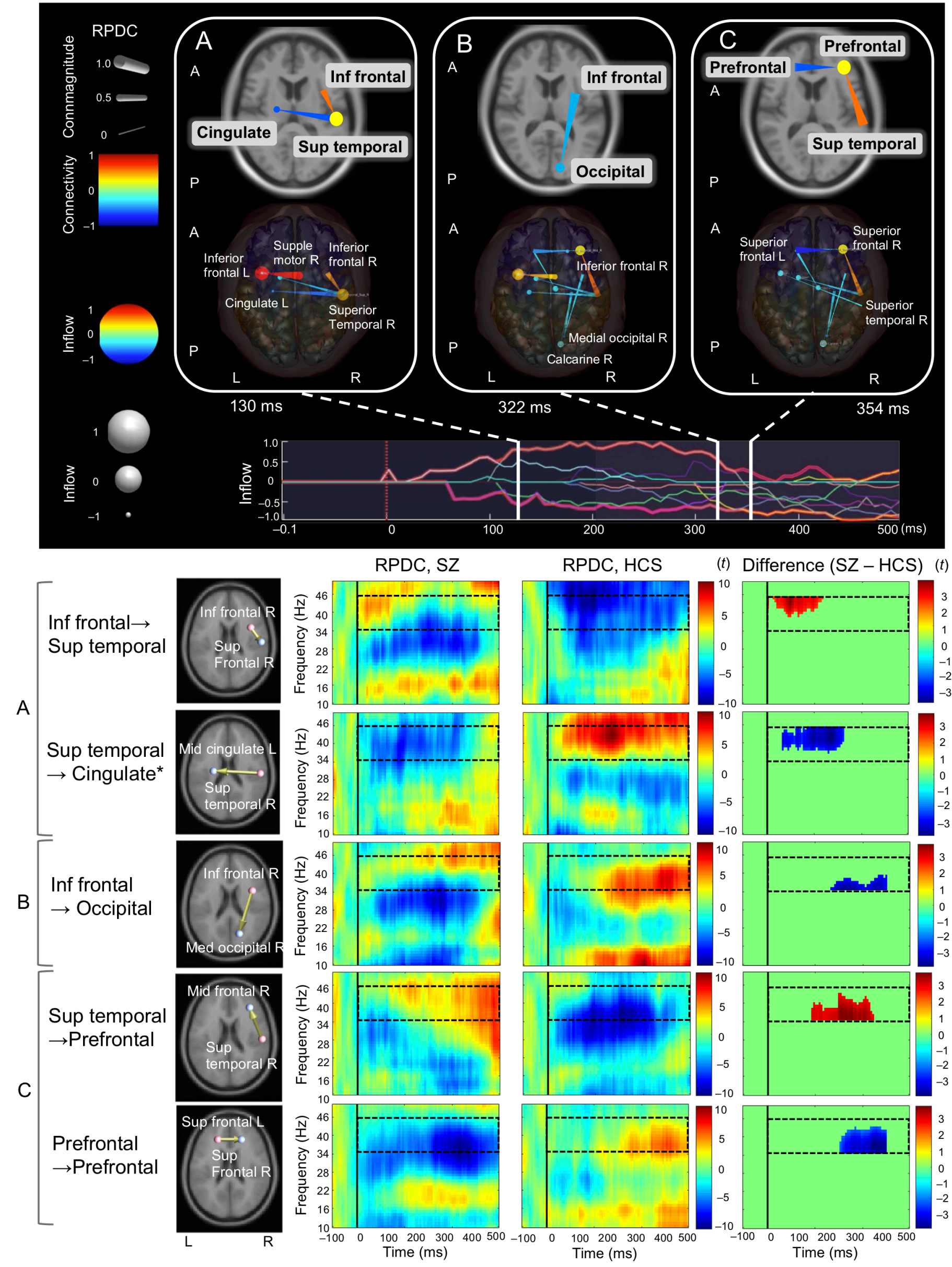
Figure 3 Koshiyama et al.

\section{A Neural networks}

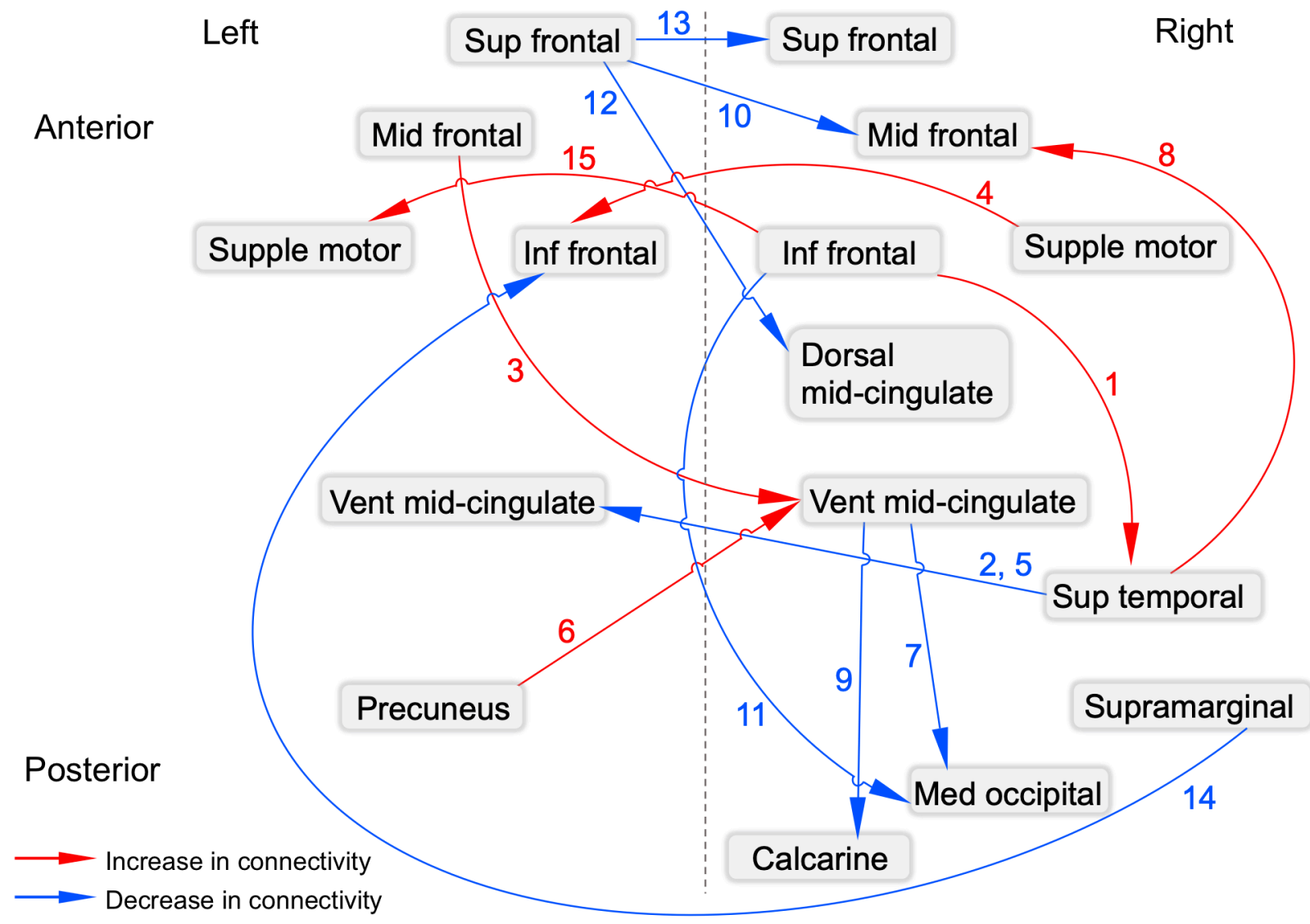

\section{B Temporal dynamics}

\section{From To}

1) Inferior frontal $R$

$\rightarrow$ Superior temporal $\mathrm{R}$

2) Superior temporal $R$

$\rightarrow$ Ventral mid-cingulate $L^{*}$

3) Middle frontal $L$

$\rightarrow$ Ventral mid-cingulate $\mathrm{R}$

4) Supple motor area $R \quad \rightarrow$ Inferior frontal $L$

5) Superior temporal $R \rightarrow$ Ventral mid-cingulate $L^{* 2}$

6) Precuneus $L$

$\rightarrow$ Ventral mid-cingulate $\mathbf{R}$

7) Ventral mid-cingulate $R \rightarrow$ Medial occipital $R$

8) Superior temporal R $\rightarrow$ Middle frontal R

9) Ventral mid-cingulate $R \rightarrow$ Calcarine $R$

10) Superior frontal $L \quad \rightarrow$ Middle frontal $R$

11) Inferior frontal $R \quad \rightarrow$ Medial occipital $R$

12) Superior frontal $L \quad \rightarrow$ Dorsal mid-cingulate $R$

13) Superior frontal $L \quad \rightarrow$ Superior frontal $R$

14) Supramarginal $R$

$\rightarrow$ Inferior frontal L

15) Inferior frontal $R$ $\rightarrow$ Supple motor area $\mathrm{L}$




Figure 4 Koshiyama et al.

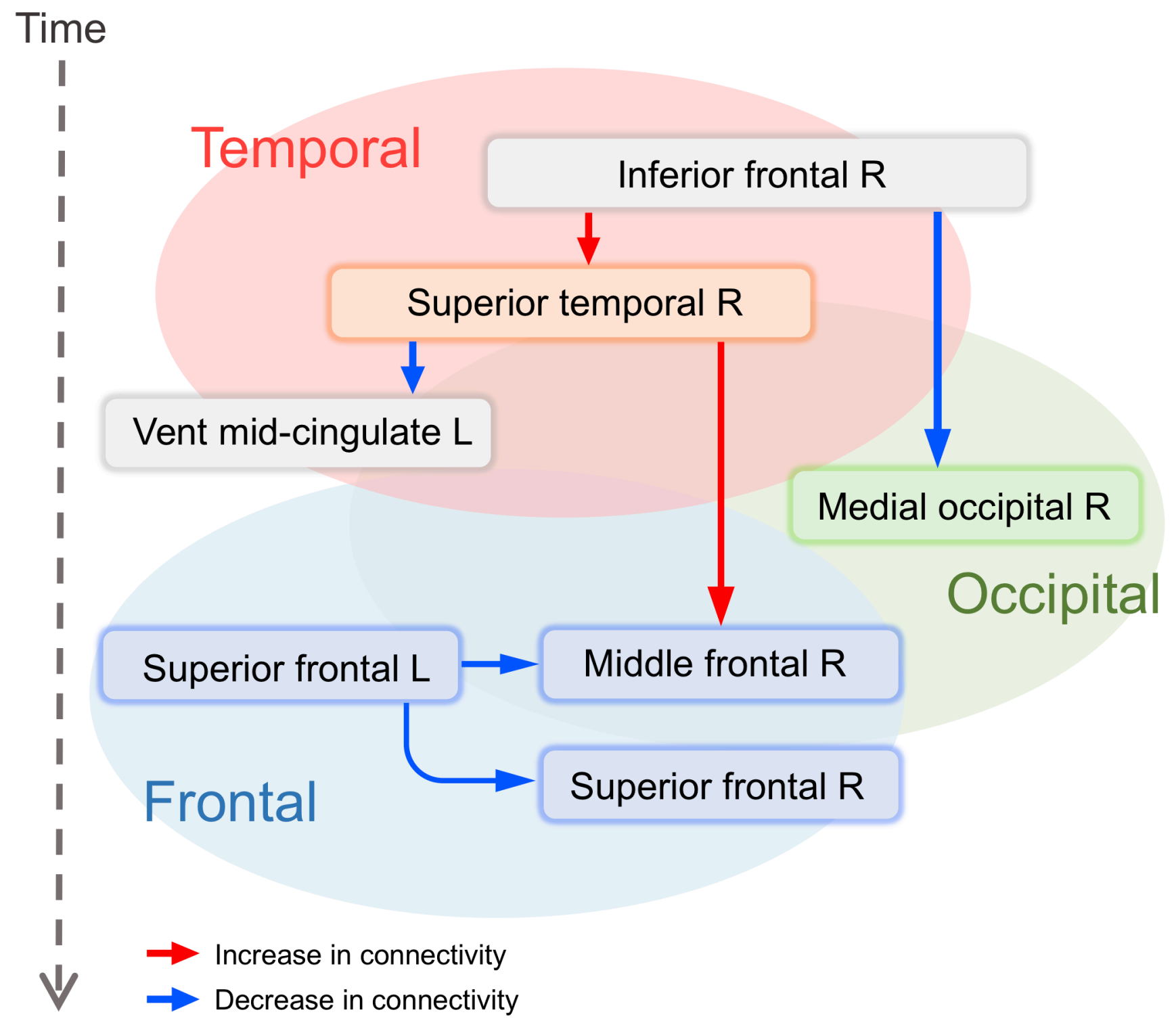




\section{Supplementary Information}

\section{Neural network dynamics underlying gamma synchronization deficits in schizophrenia}

Daisuke Koshiyama, M.D., Ph.D. ${ }^{1}$; Makoto Miyakoshi, Ph.D. ${ }^{2}$; Yash B. Joshi, M.D., Ph.D. ${ }^{1}$; Juan L. Molina, M.D. ${ }^{1}$; Kumiko Tanaka-Koshiyama, M.D. ${ }^{1}$; Joyce Sprock, B.A. ${ }^{1}$; David L. Braff, M.D. ${ }^{1}$; Neal R. Swerdlow, M.D., Ph.D. ${ }^{1}$; Gregory A. Light, Ph.D. ${ }^{1,3}$

1. Department of Psychiatry, University of California San Diego, La Jolla, CA, USA 92093-0804

2. Swartz Center for Neural Computation, University of California San Diego, La Jolla, CA, USA 92093-0559

3. VISN-22 Mental Illness, Research, Education and Clinical Center (MIRECC), VA San Diego Healthcare System, San Diego, CA, USA 92161

\section{TABLE OF CONTENTS}

\section{SUPPLEMENTARY FIGURES}

Supplementary Figure 1 Increased and decreased connectivity in schizophrenia................... 2

Supplementary Figure 2 Connectivity matrix in healthy comparison subjects......................4

Supplementary Figure 3 Connectivity matrix in schizophrenia patients.................................5

SUPPLEMENTARY MOVIES (Only title and legends)

Supplementary Movie 1 Difference of effective connectivity between schizophrenia and healthy comparison subject groups seen from an axial view.

Supplementary Movie 2 Difference of effective connectivity between schizophrenia and healthy comparison subject groups seen from a sagittal view.

Supplementary Movie 3 Effective connectivity in healthy comparison subject group seen from an axial view.

Supplementary Movie 4 Effective connectivity in healthy comparison subject group seen from a sagittal view.

Supplementary Movie 5 Effective connectivity in schizophrenia group seen from an axial view.

Supplementary Movie 6 Effective connectivity in schizophrenia group seen from a sagittal view. 
Supplementary Figure 1 Increased and decreased connectivity in schizophrenia. Abbreviations: RPDC, renormalized partial directed coherence.

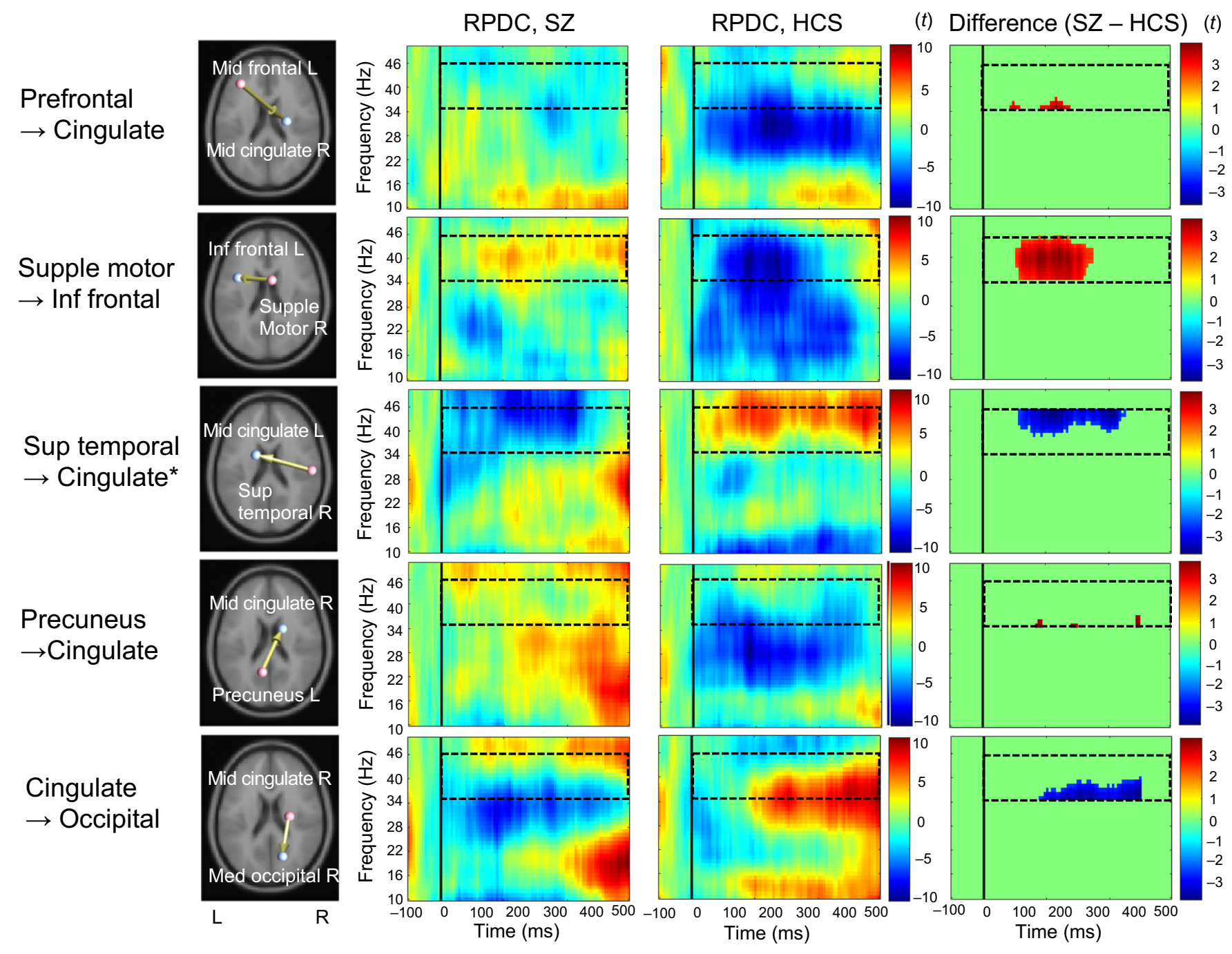


(Continued) Supplementary Figure 1

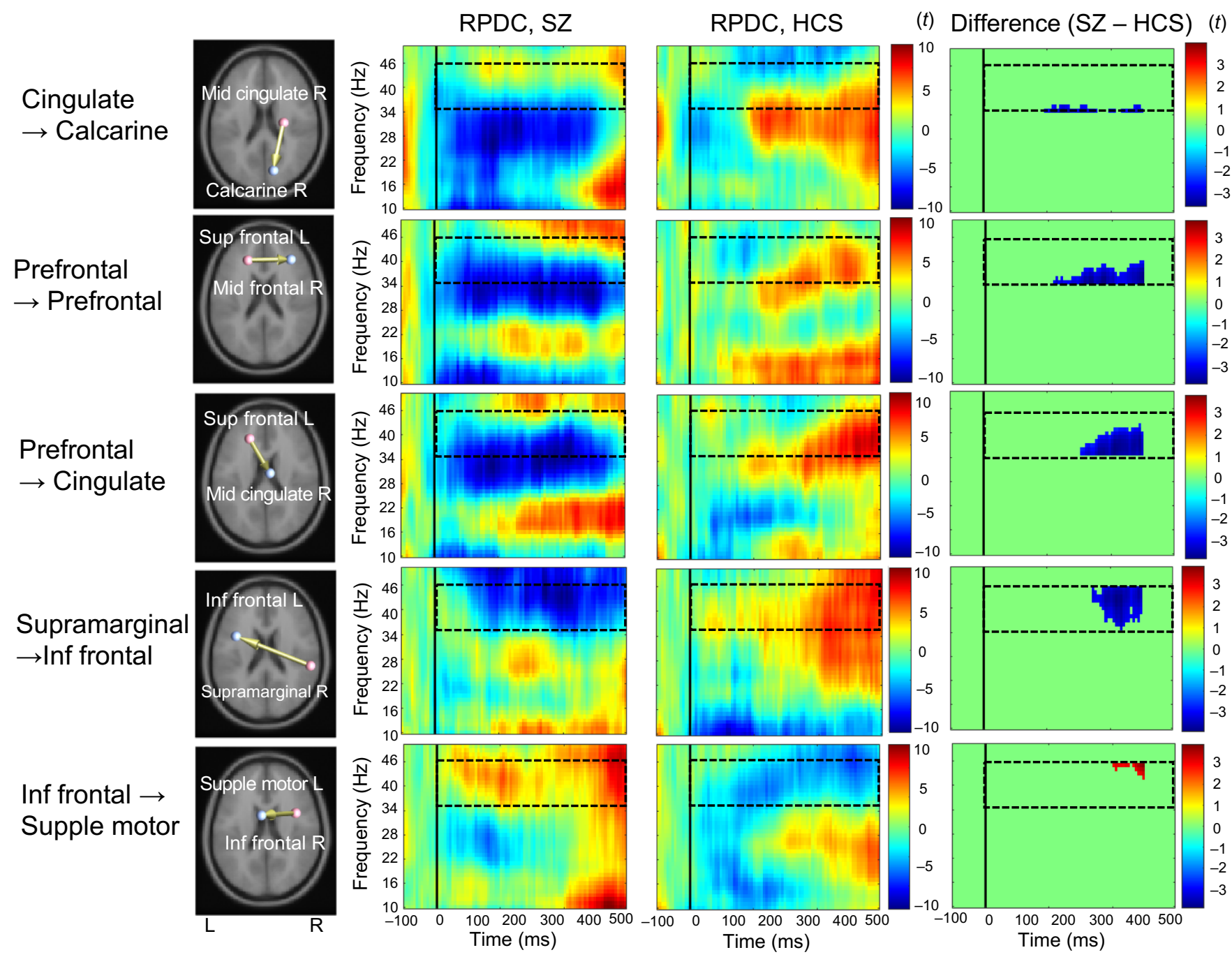

Note: Solid black line at Latency $=0$ is the stimulus onset; broken black line indicates region of interest between 35 and $45 \mathrm{~Hz}$ and between 0 and $500 \mathrm{~ms}$; 'the original label is 'upper basal' (refer to Methods and Materials section).

Abbreviations: RPDC, renormalized partial directed coherence; Mid frontal, middle frontal; Mid cingulate, middle cingulate; Supple motor area; supplementary motor area; Sup temporal; superior temporal; Inf frontal; inferior frontal; Sup temporal, superior temporal; Med occipital, medial occipital; Sup frontal, superior frontal; SZ, schizophrenia; HCS, healthy comparison subject; L, left; R, right. 
Supplementary Figure 2 Connectivity matrix of $76 \times 76$ anatomical regions of interests in healthy comparison subjects

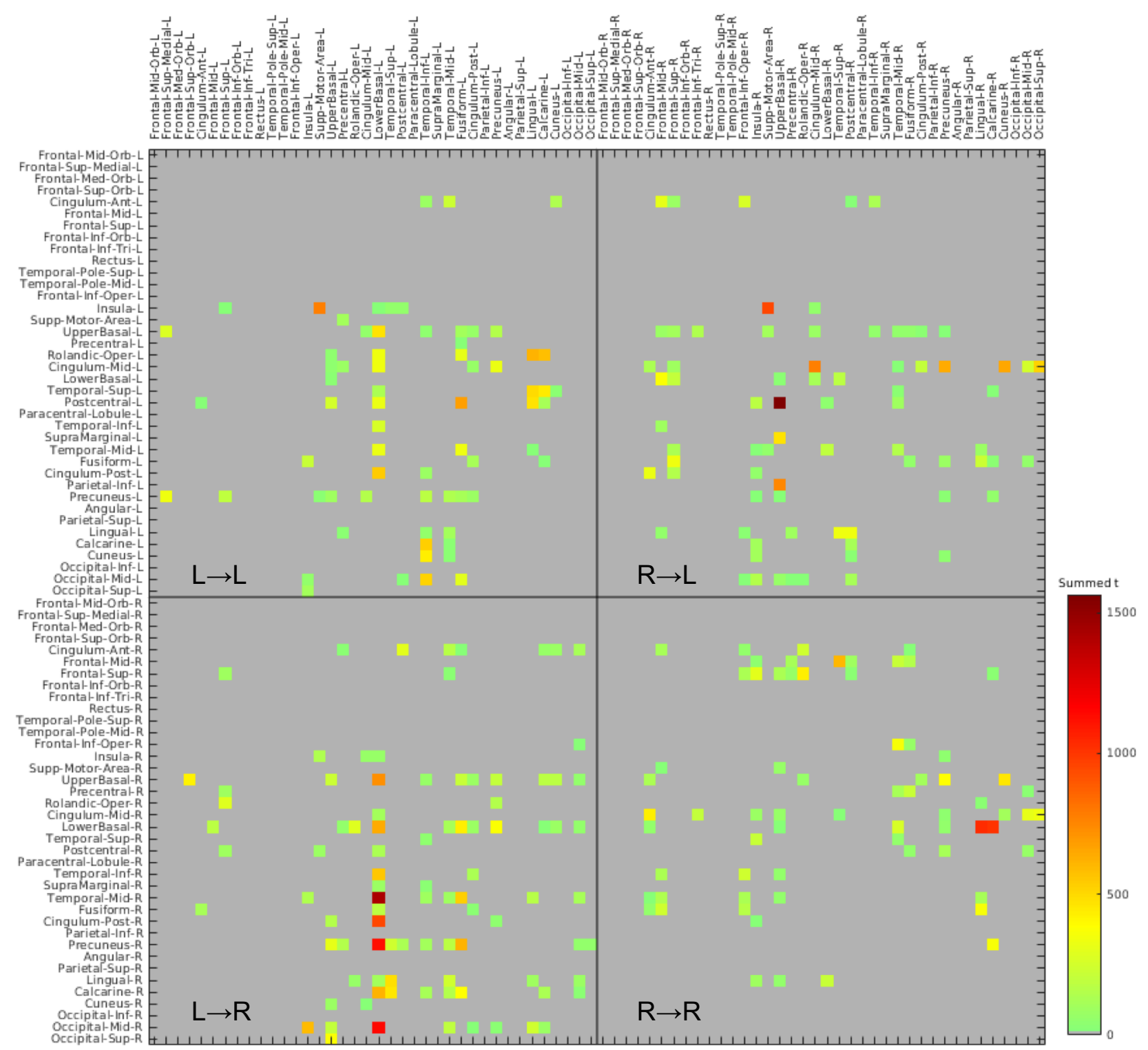


Supplementary Figure 3 Connectivity matrix of $76 \times 76$ anatomical regions of interests in schizophrenia patients

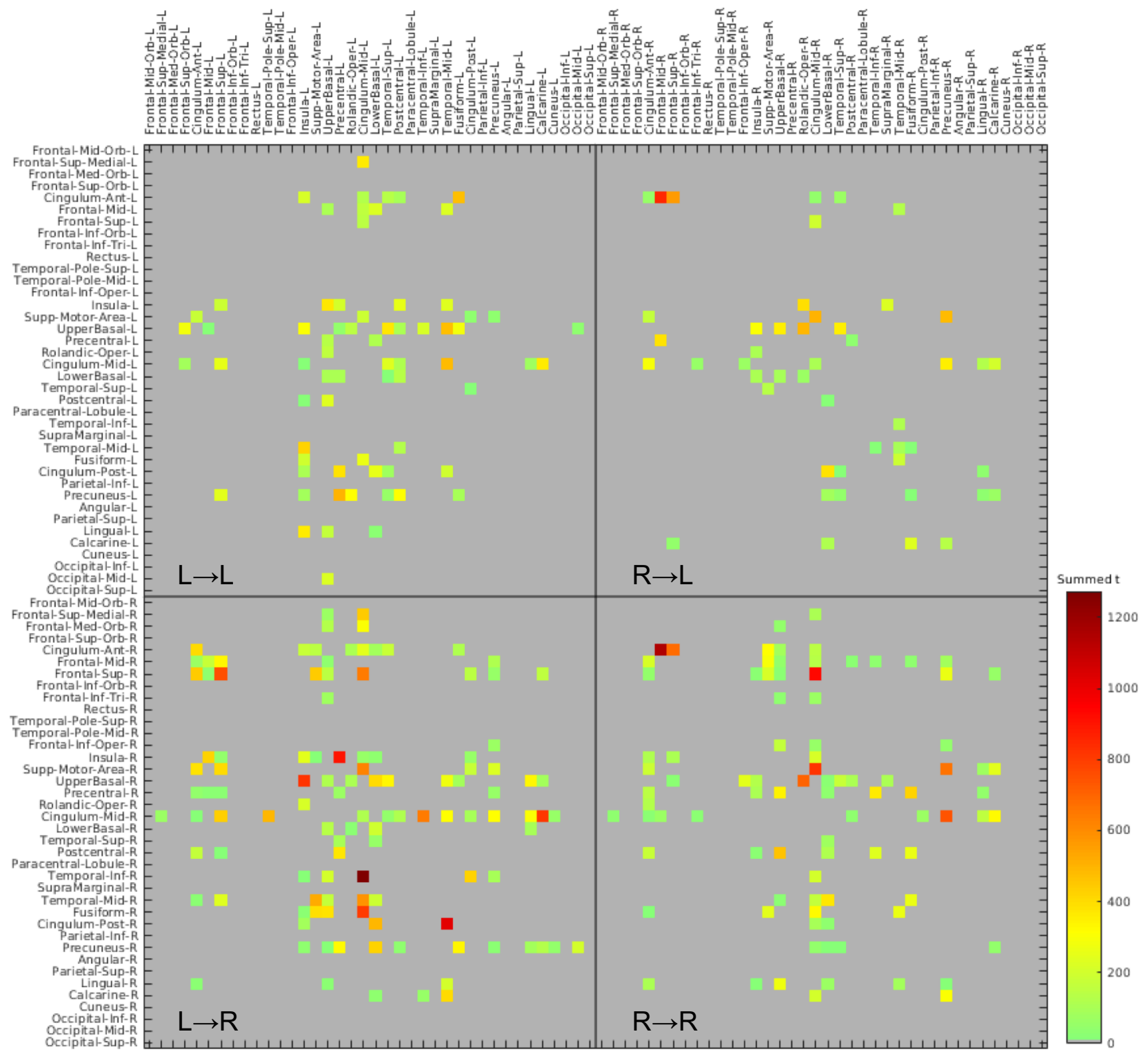




\section{SUPPLEMENTARY MOVIES (Only Notes)}

\section{Supplementary Movie 1-6}

Note: Red arrow indicates high effective connectivity (within $50 \mathrm{~ms}$ lag) and blue arrow indicates low connectivity. Connmagnitude and the color indicate amount of inflow. Sphere size and the color indicate amount of total inflow in each node. The bottom panel shows the envelope of the significant edges between 35 and $45 \mathrm{~Hz}$. Abbreviations: RPDC, renormalized partial directed coherence. 